\title{
Near-field manipulation of interparticle forces through resonant absorption, optical binding and dispersion forces
}

\author{
David S. Bradshaw and David L. Andrews* \\ School of Chemistry, University of East Anglia, Norwich Research Park, Norwich NR4 7TJ, U. K.
}

\begin{abstract}
The relative motions of two or more neutral particles, subject to optical trapping forces within a beam, are influenced by intrinsic inter-particle forces. The fundamental character of such forces is well-known and usually derives from dispersion interactions. However, the throughput of moderately intense (off-resonant) laser light can significantly modify the form and magnitude of these intrinsic forces. This optical binding effect is distinct from the optomechanical interactions involved in optical tweezers, and corresponds to a stimulated (pairwise) forward-scattering mechanism. In recent years, attention has begun to focus on optical binding effects at sub-micron and molecular dimensions. At this nanoscale, further manipulation of the interparticle forces is conceivable on the promotion of optically bound molecules to an electronic excited state. It is determined that such excitation may influence the intrinsic dispersion interaction without continued throughput of the laser beam, i.e. independent of any optical binding. Nevertheless, the forwardscattering mechanism is also affected by the initial excitation, so that both the optical binding and dispersion forces can be manipulated on input of the electromagnetic radiation. In addition, the rate of initial excitation of either molecule (or any energy transfer between them) may be influenced by an off-resonant input beam which, thus, acts as an additional factor in the modification of the interparticle force. A possible experimental set-up is proposed to enable the measurement of such changes in the interparticle coupling.
\end{abstract}

Keywords: optical binding, dispersion forces, optical manipulation, excited states, interparticle interactions, near field, nanophotonics, quantum electrodynamics

\section{INTRODUCTION}

Many fundamental optical forces that originate in particle couplings fundamentally rely on virtual photons to mediate the electromagnetic coupling. A well-known example is the dispersion interaction that operates between electrically neutral, non-polar particles. ${ }^{1-6}$ On irradiating any such interacting particle pair with a throughput laser beam, the phenomenon of optical binding may, however, override the intrinsic dispersion interaction. This all-optical process entails the pairwise elastic forward-scattering of a passive (off-resonant laser) beam, without any net absorption or stimulated emission, leading to a modification to the dispersion interaction. This phenomenon has increasingly been advocated as a tool for the optical manipulation and configuration of particles, ${ }^{7-16}$ and optically induced arrays have been observed experimentally. ${ }^{17,18}$ In conventional studies, all such particles are in their ground state. To broaden the scope, we now report the results of an analysis of optical binding removing this restriction, built on the premise that certain particles are optically excited. This proves to enable the engineering of a change in magnitude in the optical binding force. Excitation of the particles is achieved by direct absorption of a suitably resonant laser beam, or resonance energy transfer between particles. Starting with a focus on conventional optical interactions, a quantum formulation corresponding to optical binding and the dispersion interaction is presented for nanoscale application. We then address the situation in which either or both nanoparticles, in each pair that undergo optical binding, are electronically excited. An analysis of how the required resonant absorption or resonant energy transfer may also be affected by the off-resonant laser beam is then given. A discussion section then concludes the paper. 


\section{CONVENTIONAL OPTICAL FORCES}

\subsection{Optical binding}

To adequately represent all the quantum features of an optical binding mechanism, the Power-Zienau-Woolley approach is employed. ${ }^{19}$ Using this framework, the multipolar Hamiltonian, $H$, for a pair of nanoparticles $A$ and $B$ is given by;

$$
H=\sum_{\xi=A, B} H_{\mathrm{part}}(\xi)+\sum_{\xi=A, B} H_{\mathrm{int}}(\xi)+H_{\mathrm{rad}}
$$

where $H_{\text {part }}$ is the Hamiltonian for nanoparticle $\xi$ and $H_{\text {rad }}$ denotes the energy operator for the radiation field. The Hamiltonian $H_{\text {int }}$ represents the interaction of the field with $\xi$ and, within the electric dipole approximation, is given by the following;

$$
H_{\text {int }}(\xi)=-\varepsilon_{0}^{-1} \sum_{\xi} \boldsymbol{\mu}(\xi) \cdot \mathbf{d}^{\perp}\left(\mathbf{R}_{\xi}\right)
$$

with $\boldsymbol{\mu}(\xi)$ and $\mathbf{R}_{\xi}$ as the electric-dipole moment operator and the position vector, respectively. The operator $\mathbf{d}^{\perp}\left(\mathbf{R}_{\xi}\right)$ represents the transverse electric displacement field of the radiation. General results for the optical binding forces between a pair of neutral nanoparticles $A$ and $B$ can be determined from an expression for the distance-dependent energy shift, $\Delta E$, which in turn is obtained by the application of fourth-order perturbation theory;

$$
\Delta E=\operatorname{Re}\left\{\sum_{t, s, r} \frac{\left\langle i\left|H_{\text {int }}\right| t\right\rangle\left\langle t\left|H_{\text {int }}\right| s\right\rangle\left\langle s\left|H_{\text {int }}\right| r\right\rangle\left\langle r\left|H_{\text {int }}\right| i\right\rangle}{\left(E_{i}-E_{t}\right)\left(E_{i}-E_{s}\right)\left(E_{i}-E_{r}\right)}\right\},
$$

where $|i\rangle$ is the unperturbed system state in which both $A$ and $B$ are in their individual electronic ground states; $|r\rangle,|s\rangle$ and $|t\rangle$ are virtual system states, and $E_{n}$ is the energy of state $|n\rangle$. The system states can be written in the form;

$$
|n\rangle=\left|\operatorname{part}_{n}\right\rangle\left|\operatorname{rad}_{n}\right\rangle \equiv\left|\operatorname{part}_{n} ; \operatorname{rad}_{n}\right\rangle \text {, }
$$

with $\left|\operatorname{part}_{n}\right\rangle$ and $\left|\operatorname{rad}_{n}\right\rangle$ defining the status of all nanoparticle and radiation states, respectively. The laser-induced interaction entails the absorption of a 'real' (laser throughput) photon at one nanoparticle and the stimulated emission of a 'real' photon at the other, with a virtual photon acting as mediator between them; this results in elastic forwardscattering of the passive beam as shown by figure 1. The nanoparticles and the throughput radiation suffer no overall change in quantum state. Using the implied summation convention for repeated Cartesian tensor subscript indices, as shown previously, the short-range result for $\Delta E$ emerges as; ${ }^{7}$

$$
\Delta E=\left(\frac{I}{\varepsilon_{0} c}\right) e_{i}^{(\eta)} \bar{e}_{l}^{(\eta)} \operatorname{Re}\left(\alpha_{i j}^{00(A)}(\omega) V_{j k}(q, \mathbf{R}) \alpha_{k l}^{00(B)}(\omega)\right),
$$

where $I$ is the intensity of the passive beam, $\hbar c q \equiv \hbar \omega$ and $\mathbf{e}^{(\eta)}$ are (respectively) the energy per photon and polarization of the input beam, $\alpha_{i j}^{00\left(\xi_{m}\right)}(\omega)$ represents the static polarizability tensor at circular optical frequency $\omega$, and $V_{j k}$ signifies the electric dipole-electric dipole interaction tensor, whose components are strongly dependent on the direction and magnitude of the intermolecular displacement vector: details are given elsewhere. ${ }^{20,21}$ The optical force is determined from equation (5) via $\mathbf{F}=-\partial \Delta E / \partial \mathbf{R}$, from which the magnitude of the optical binding force, in the short-range (nanoscale) region, is found to be dominated by a term with $R^{-4}$ distance dependence. 


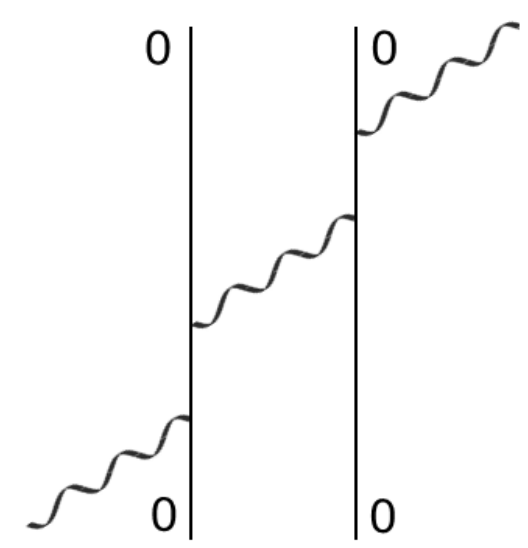

(a)

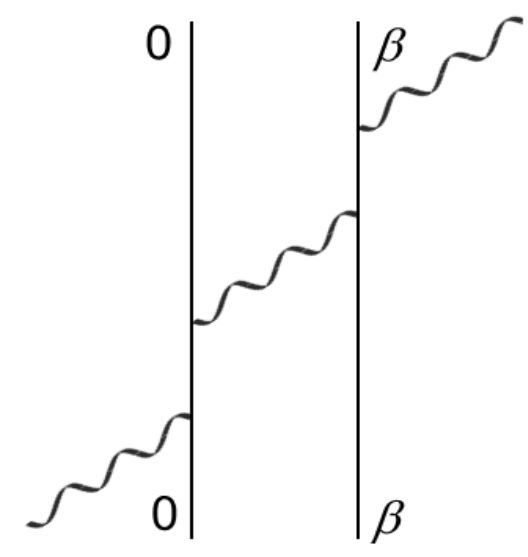

(c)

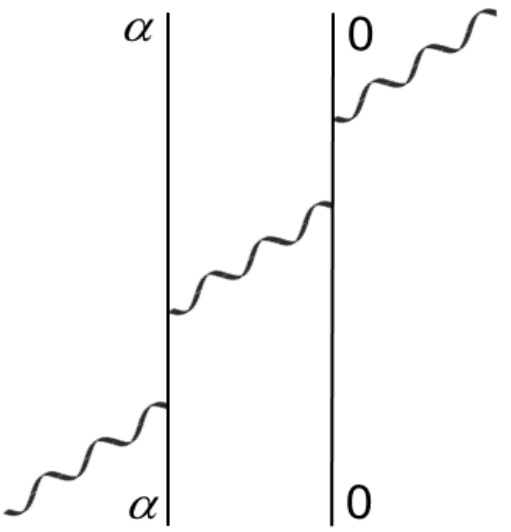

(b)

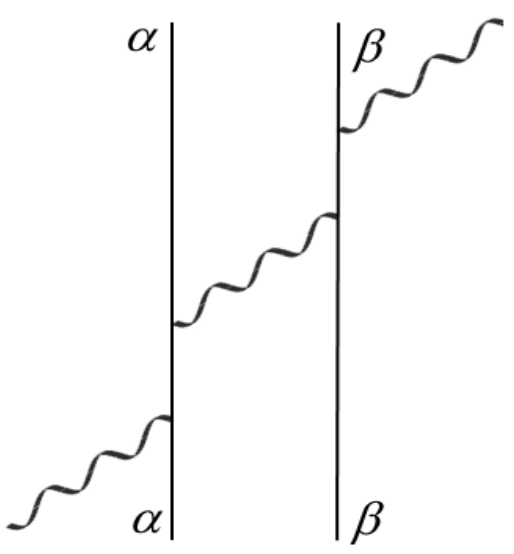

(d)

Figure 1. Feynman diagram for optical binding, the two vertical lines denote molecules ( $A$ on the left and $B$ the right), the wavy lines photons: (a) conventional binding, where the two particles begin and end in their ground state; $(b)-(d)$ alternative configurations where either (or both) particles are excited (states denoted by $\alpha$ and $\beta$ ) giving a different optical binding force to the conventional case.

\subsection{Dispersion interaction}

In the quantum formulation, dispersion interactions entail two virtual photons that again produce four particle-photon couplings. Derived from which the following general expression is ascertained, in the short-range region; ${ }^{3}$

$$
\Delta E=-\sum_{s, t} \frac{\mu_{i}^{0 s(A)} V_{i j}(0, \mathbf{R}) \mu_{j}^{0 t(B)} \mu_{k}^{s 0(A)} V_{k l}(0, \mathbf{R}) \mu_{l}^{t 0(B)}}{E_{s 0}^{A}+E_{t 0}^{B}},
$$

where $\mu_{i}$ is a transition dipole moment, and $E_{s 0}=E_{s}-E_{0}$ etc.; moreover, $s$ and $t$ are intermediate virtual states of nanoparticles $A$ and $B$, respectively. On applying an isotropic average to equation (6) the London formula arises, i.e.; 


$$
\Delta E=-\frac{1}{24 \pi^{2} \varepsilon_{0}^{2} R^{6}} \sum_{s, t} \frac{\left|\mu^{0 s(A)}\right|^{2}\left|\mu^{0 t(B)}\right|^{2}}{E_{s 0}^{A}+E_{t 0}^{B}} .
$$

The energy differences $E_{s 0}^{A}$ and $E_{t 0}^{B}$ always produce positive values, invariably creating a negative quantity for the result of equation Error! Reference source not found.. From the relation between force and potential energy, this physically corresponds to an attractive interaction.

\section{MODIFYING OPTICAL BINDING}

In the previous analysis, the optical binding theory was based on the standard assumption that both nanoparticles are in their ground state. In this section the investigation turns to cases where either (or both) nanoparticles are excited, via direct photo-excitation or resonance energy transfer. Let us suppose that the dispersion interaction is negligible compared to the optical binding; although it is interesting that, in certain circumstances, the denominator of equation (7) may be negative if either nanoparticle is excited - denoting that the dispersion force is not always attractive under such conditions. ${ }^{22}$ Unlike Section 2, where optical binding operates in isolation, we now consider the phenomenon in the context of resonant absorption or resonance energy transfer. The observables for these phenomena remain independent and do not - indeed cannot - together forge a new mechanism. First, we compare the optical binding potential when $A$ is excited, with the conventional case, so that;

$$
\Delta E(\alpha, 0)-\Delta E(0,0) \sim \alpha_{i j}^{\alpha \alpha(A)}(\omega) V_{j k}(q, \mathbf{R}) \alpha_{k l}^{00(B)}(\omega)-\alpha_{i j}^{00(A)}(\omega) V_{j k}(q, \mathbf{R}) \alpha_{k l}^{00(B)}(\omega),
$$

which follows from equation (5), and the explicit form of the generalized polarizability tensor is given by;

$$
\alpha_{i j}^{n n(\xi)}(\omega)=\sum_{s}\left\{\frac{\mu_{i}^{n s(\xi)} \mu_{j}^{s n(\xi)}}{E_{s a}-\hbar \omega}+\frac{\mu_{j}^{n s(\xi)} \mu_{i}^{s n(\xi)}}{E_{s a}+\hbar \omega}\right\},
$$

where $n$ denotes $\xi$ in either a ground or excited state (i.e. $\alpha$ or $\beta$ ), depending on the particle to which the formula is applied. If the input photon is chosen so that a factor $\delta=E_{\alpha 0}-\hbar \omega$ is very small, but outside of the resonance absorption range, and a two-level system is employed (which means that $s$ corresponds to either a ground or excited state), equation (8) becomes;

$$
\Delta E(\alpha, 0)-\Delta E(0,0) \sim-\frac{2 \mu_{i}^{0 \alpha(A)} \mu_{j}^{\alpha 0(A)} V_{j k}(q, \mathbf{R}) \mu_{k}^{0 \beta(B)} \mu_{l}^{\beta 0(B)}}{\delta^{2}} .
$$

Here, $E_{\alpha 0} \approx E_{\beta 0}$ and only the leading term - which greatly dominates all the others - is shown. Inserting the short-range form of the $\mathbf{V}$ tensor into equation (10), we obtain the result;

$$
\Delta E(\alpha, 0)-\Delta E(0,0)=-\left(\frac{I}{2 \pi c \varepsilon_{0}^{2} R^{3}}\right) e_{i}^{(\eta)} \bar{e}_{l}^{(\eta)} \operatorname{Re}\left(\frac{\mu_{i}^{0 \alpha(A)} \mu_{j}^{\alpha(A)}\left(\delta_{j k}-3 \hat{R}_{j} \hat{R}_{k}\right) \mu_{k}^{0 \beta(B)} \mu_{l}^{\beta 0(B)}}{\delta^{2}}\right) .
$$

If the excitation is then transferred from $A$ to $B$, via a resonance energy transfer step, the change in optical binding potential is given as;

$$
\Delta E(0, \beta)-\Delta E(\alpha, 0)=0,
$$

under the constraints that have been applied; in general the result will be non-zero. 


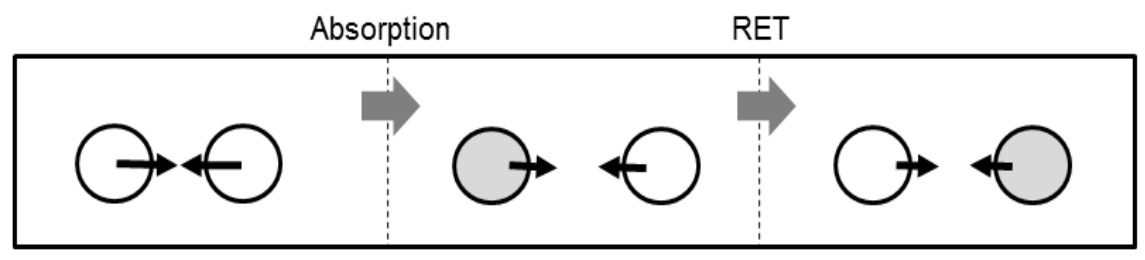

(a)

(b)

$(c)$

Figure 2. Representation of optical binding for: $(a) A$ and $B$ both in their ground states (unfilled circles), $(b) A$ in an excited state (filled circle) with $B$ remaining unexcited, and $(c) A$ unexcited and $B$ excited. The successive states are achievable via the sequential optical process, shown horizontally across the diagram, of one-photon absorption (first block arrow) followed by resonance energy transfer (second block arrow). The black arrows on the nanoparticles denote changes in the optical binding force; the rate of absorption and energy transfer may also be modified by the presence of the off-resonant beam (not shown).

Figure 2 visually illustrates, and serves to reinforce the physical import of the above calculations; it shows optical binding between two nanoparticles. The depiction of the pair on the left involves both nanoparticles in their ground state. Moving across the diagram, $A$ is excited through one-photon absorption (RET from a nearby non-interacting particle is also a plausible source) - enabling the possibility that the pair potential may be modified as determined earlier. A process of resonance energy transfer between the pair is then represented on the diagram as we move to the right-hand side. In the presence of this off-resonant beam the rate of both resonant absorption and resonance energy transfer may be modified; this subject is briefly outlined in the following Section.

\section{LASER-ASSISTED ABSORPTION AND ENERGY TRANSFER}

\subsection{Laser-modified light absorption}

In addition to optical binding, passive laser control may also enable higher-order effects to occur, based on the accommodation of other fundamental photonic processes. This can, for example, be achieved by engagement of the off-resonant beam, resulting in nonlinear interactions. We first examine these effects in application to resonant photon absorption; the mechanism is shown in Fig. 3(a). It is considered self-evident that photon absorption of light by nanoparticles occurs through individual photons of an appropriate wavelength resonantly interacting with the individual particles. A corollary to this premise is that photons of a wavelength outside the range that the particle directly absorbs generally play no part in the absorption of other, suitably resonant photons. The possibility of this higher-order effect proves that this conjecture is not totally correct, since the input of a passive beam of sufficient intensity may alter the rate of photon absorption. The full detail of this process is described in ongoing work and is of a similar nature to that which may operate in molecular fluorescence, as we have shown in other recent work. ${ }^{23-25}$

\subsection{Laser-assisted resonance energy transfer}

In the case of RET, an off-resonant laser beam may also enhance (or diminish) the process through a conferred optical response known as laser-assisted resonance energy transfer. ${ }^{26}$ This again involves elastic forward-scattering of the passive beam, in an optically catalytic sense without any net absorption or stimulated emission - as illustrated by Fig. 3(b). Despite the apparent similarity to the mechanism for optical binding, this interaction is subtly dissimilar since the initial and final system states now differ: the excitation initially on particle $A$ moves to particle $B$. Having the ability to exert optical control over the migration of energy provides a possible basis for all-optical switching in an adapted form of laser-assisted energy transfer, where the spontaneous process is completely disabled. ${ }^{27-30}$ This can be achieved by engineering conditions of transition moment orthogonality, $\boldsymbol{\mu}^{0 \alpha(A)} \perp \mathbf{R} \perp \boldsymbol{\mu}^{\beta 0(B)}$, which results in spontaneous energy transfer being excluded by geometry. Alternatively, spontaneous transfer may be inhibited when the electronic transition in either the donor or acceptor is one-photon forbidden, but two-photon allowed. In either case, switching action induced by the transport of energy is activated only when the passive beam is 'on'. The parallel-processing capability of such a 
device introduces a variety of applications. For example, it indicates that pixel-based images, written by donor excitation, might be controllably transferred with high fidelity to an acceptor film. Such a device offers a potential for data buffer and other optical informatics operations. ${ }^{30}$

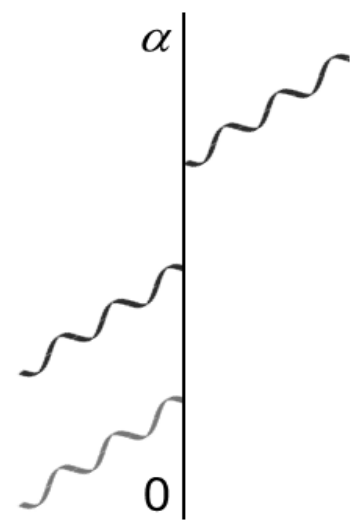

(a)

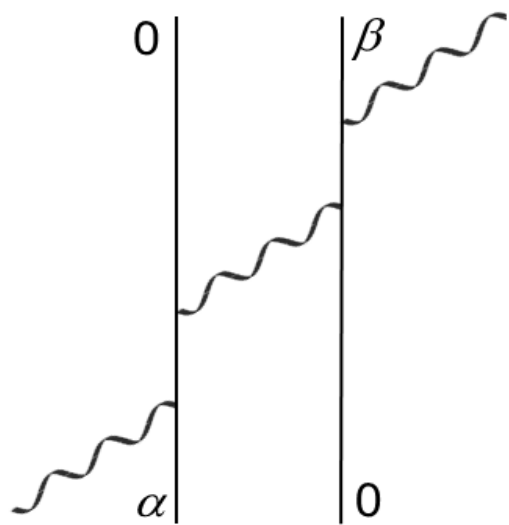

(b)

Figure 3. Feynman diagram for ( $a$ ) laser-modified light absorption and $(b)$ laser-assisted resonance energy transfer. As figure 1, except that the grey wavy line (lower left) denotes an absorbing photon from a resonant beam, rather than the passive laser.

\section{DISCUSSION}

Our research has shown that on excitation of either interacting particle, a significant change in the potential energy of optical binding is possible. This contrasts with conventional optical binding, where both particles are normally in their ground state. Detailed calculations, based on the equations presented here and assuming typical values for the material parameters, show that the intensity regime of the input laser needs to be only around $10^{10}-10^{12} \mathrm{~W} \mathrm{~cm}^{-2}$ for practical observation of a change in optical force. Such levels of irradiance are well within the range of current pulsed laser systems.

It is not hard to devise experimental set-ups to pursue investigations of these effects. The surface functionalization of nanoparticles such as carbon nanotubes might be one way to produce particles readily suited for optical excitation at an amenable wavelength. In an array of such nanoparticles, conventionally optically bound by an off-resonant laser beam, pulses of the resonant wavelength light from a secondary laser source will enable a proportion of the particles to be electronically excited. The ensuing changes in local potential energy of interaction with neighboring particles should then be manifest in a local rearrangement of the array structure that will be easily detected.

\section{ACKNOWLEDGMENTS}

The authors would like to thank the Leverhulme Trust for funding this research. 


\section{REFERENCES}

[1] Cohen, A. E. and Mukamel, S., "Resonant enhancement and dissipation in nonequilibrium van der Waals forces," Phys. Rev. Lett. 91, 233202 (2003).

[2] Obrecht, J. M., Wild, R. J., Antezza, M., Pitaevskii, L. P., Stringari, S. and Cornell, E. A., "Measurement of the temperature dependence of the Casimir-Polder force," Phys. Rev. Lett. 98, 233202 (2007).

[3] Andrews, D. L., Bradshaw, D. S., Leeder, J. M. and Rodríguez, J., "Dynamics of the dispersion interaction in an energy transfer system,” Phys. Chem. Chem. Phys. 10, 5250-5255 (2008).

[4] Buhmann, S. Y. and Scheel, S., "Thermal Casimir versus Casimir-Polder forces: Equilibrium and nonequilibrium forces," Phys. Rev. Lett. 100, 253201 (2008).

[5] Sherkunov, Y., "Dispersion interaction between two atoms out of thermal equilibrium with external electromagnetic fields," Phys. Rev. A 79, 244107 (2009).

[6] Rodríguez, J. and Salam, A., "On the role of dissipation on the Casimir-Polder potential between molecules in dielectric media,” J. Chem. Phys. 133, 164501 (2010).

[7] Bradshaw, D. S. and Andrews, D. L., "Optically induced forces and torques: Interactions between nanoparticles in a laser beam," Phys. Rev. A 72, 033816 (2005).

[8] Grzegorczyk, T. M., Kemp, B. A. and Kong, J. A., "Stable optical trapping based on optical binding forces," Phys. Rev. Lett. 96, 113903 (2006).

[9] Guillon, M., Moine, O. and Stout, B., "Longitudinal optical binding of high optical contrast microdroplets in air," Phys. Rev. Lett. 96, 143902 (2006).

[10] Salam, A., "Intermolecular interactions in a radiation field via the method of induced moments," Phys. Rev. A 73, 013406 (2006).

[11] Karásek, V. and Zemánek, P., "Analytical description of longitudinal optical binding of two spherical nanoparticles,” J. Opt. A: Pure Appl. Opt. 9, S215-S220 (2007).

[12] Marchington, R. F., Mazilu, M., Kuriakose, S., Garcés-Chávez, V., Reece, P. J., Krauss, T. F., Gu, M. and Dholakia, K., "Optical deflection and sorting of microparticles in a near-field optical geometry," Opt. Express 16, 3712-3726 (2008).

[13] Haefner, D., Sukhov, S. and Dogariu, A., "Conservative and nonconservative torques in optical binding," Phys. Rev. Lett. 103, 173602 (2009).

[14] Taylor, J. M. and Love, G. D., “Optical binding mechanisms: A conceptual model for Gaussian beam traps,” Opt. Express 17, 15381-15389 (2009).

[15] Čižmár, T., Dávila Romero, L. C., Dholakia, K. and Andrews, D. L., "Multiple optical trapping and binding: New routes to self-assembly," J. Phys. B: At. Mol. Opt. Phys. 43, 102001 (2010).

[16] Dholakia, K. and Zemanek, P., “Gripped by light: Optical binding,” Rev. Mod. Phys. 82, 1767-1791 (2010).

[17] Mohanty, S. K., Andrews, J. T. and Gupta, P. K., "Optical binding between dielectric particles," Opt. Express 12 , 2746-2753 (2004).

[18] Mellor, C. D., Fennerty, T. A. and Bain, C. D., "Polarization effects in optically bound particle arrays," Opt. Express 14, 10079-10088 (2006).

[19] Woolley, R. G., "Gauge invariance in non-relativistic electrodynamics," Proc. R. Soc. London, Ser. A 456, $1803-$ 1819 (2000).

[20] Daniels, G. J., Jenkins, R. D., Bradshaw, D. S. and Andrews, D. L., "Resonance energy transfer: The unified theory revisited," J. Chem. Phys. 119, 2264-2274 (2003).

[21] Andrews, D. L. and Bradshaw, D. S., "Virtual photons, dipole fields and energy transfer: a quantum electrodynamical approach," Eur. J. Phys. 25, 845-858 (2004).

[22] Bradshaw, D. S. and Andrews, D. L., "Interparticle interactions: Energy potentials, energy transfer, and nanoscale mechanical motion in response to optical radiation," J. Phys. Chem. A 117, 75-82 (2013).

[23] Bradshaw, D. S. and Andrews, D. L., "Mechanism for optical enhancement and suppression of fluorescence," J. Phys. Chem. A 113, 6537-6539 (2009).

[24] Bradshaw, D. S. and Andrews, D. L., “All-optical control of molecular fluorescence,” Phys. Rev. A 81, 013424 (2010).

[25] Leeder, J. M., Bradshaw, D. S. and Andrews, D. L., "Laser-controlled fluorescence in two-level systems,” J. Phys. Chem. B 115, 5227-5233 (2011). 
[26] Allcock, P., Jenkins, R. D. and Andrews, D. L., “Laser-assisted resonance-energy transfer,” Phys. Rev. A 6102, 023812 (2000).

[27] Andrews, D. L., “Optically switched energy transfer: Twin-beam off-resonance control,” Phys. Rev. Lett. 99, 023812 (2007).

[28] Bradshaw, D. S. and Andrews, D. L., "Optically controlled resonance energy transfer: Mechanism and configuration for all-optical switching," J. Chem. Phys. 128, 144506 (2008).

[29] Bradshaw, D. S. and Andrews, D. L., "All-optical switching based on controlled energy transfer between nanoparticles in film arrays," J. Nanophoton. 3, 031503 (2009).

[30] Bradshaw, D. S. and Andrews, D. L., "All-optical switching between quantum dot nanoarrays," Superlatt. Microstruct. 47, 308-313 (2010).

*david.andrews@physics.org 